\title{
BAUDELAIRE ET LA « HAINE DU VÉGÉTAL »
}

BAUDELAIRE AND "HATE OF VEGETABLE"

Aurélia Cervoni

Université Paris Sorbonne

Paris, França

ORCID 0000-0002-4214-3937

\section{Résumé}

Dans les premières lignes d'Anywhere out of the world, Baudelaire prête aux habitants de Lisbonne la " haine du végétal ». Cet article se propose d'éclairer le sens de cette formule d'un point de vue historique, esthétique et polémique, en se fondant sur l'œuvre et la correspondance de Baudelaire, ainsi que sur l'intertexte romantique (Théophile Gautier, Pétrus Borel).

Mots-clés : Poésie moderne, romantisme, panthéisme, lycanthropie.

\begin{abstract}
In the first lines of Anywhere out of the world, Baudelaire dreams to go to Lisbon, where people "hate vegetation". Based on baudelairian and romantic intertext (Théophile Gautier, Petrus Borel), this article aims to shed light on the meaning of this expression from an historical, aesthetical and polemical point of view.
\end{abstract}

Keywords: Modern poetry, romanticism, pantheism, lycanthropy.

\section{Resumo}

Nas primeiras linhas de Anywhere out of the world, Baudelaire atribui aos habitantes de Lisboa o "ódio do vegetal". O objetivo deste artigo é esclarecer o significado dessa fórmula de um ponto de vista histórico, estético e polêmico, baseado na obra e na correspondência de Baudelaire, bem como no intertexto romântico (Théophile Gautier, Pétrus Borel).

Palavras-chave: Poesia Moderna, Romantismo, Panteísmo, Licantropia. 
Dans Any where out of the world, où il envisage d'émigrer à Lisbonne pour tromper le mal de vivre, l'auteur du Spleen de Paris prête aux habitants de la ville la " haine du végétal » :

Dis-moi, mon âme, pauvre âme refroidie, que penserais-tu d'habiter Lisbonne? Il doit y faire chaud, et tu t'y ragaillardirais comme un lézard. Cette ville est au bord de l'eau ; on dit qu'elle est bâtie en marbre, et que le peuple y a une telle haine du végétal, qu'il arrache tous les arbres. Voilà un paysage selon ton goût ; un paysage fait avec la lumière et le minéral, et le liquide pour les réfléchir! (BAUDELAIRE, 1975, t. I, p. 356)

Le poète amplifie les observations des voyageurs de son temps, sensibles au charme de la capitale portugaise : bâtie en amphithéâtre sur les rives du Tage, la ville se distingue par ses églises, recouvertes d'une «belle pierre de marbre [...] blanche, polie, brillante ${ }^{1}$ (MERSON, 1857, p. 90), et par ses maisons " en partie revêtues de plaques de faïence " (VOGEL, 1860, p. 472). Tout en soulignant l'élégance des avenues, quelques descriptions regrettent l'absence de " grands arbres » et de leurs " frais ombrages " (VOGEL, 1860, p. 477). Nous n'avons retrouvé aucune allusion à une éventuelle campagne d'arrachage des arbres dans la ville.

La critique a relevé les liens intertextuels qui unissent Any where out of the world et Rêve parisien, dans Les Fleurs du Mal² :

De ce terrible paysage,

Tel que jamais mortel n'en vit,

Ce matin encore l'image,

Vague et lointaine, me ravit.

Le sommeil est plein de miracles!

Par un caprice singulier,

J'avais banni de ces spectacles

Le végétal irrégulier,

Et, peintre fier de mon génie,

Je savourais dans mon tableau

L'enivrante monotonie

Du métal, du marbre et de l'eau.

1 Voir aussi Alfred Germond de Lavigne, Itinéraire descriptif, historique et artistique de l'Espagne et $d u$ Portugal, Paris, Hachette, 1859, p. 760 ; Auguste Lannau-Rolland, Nouveau guide général du voyageur en Espagne et en Portugal, Paris, Garnier, 1864, p. 466 et 473 ; entrée "Lisbonne " du Grand dictionnaire universel du XIXe siècle de Larousse, t. X, 1873, p. 559.

2 Voir par exemple Felix Leakey, Baudelaire and Nature, Manchester University Press, 1969, p. 291-294. 
Babel d'escaliers et d'arcades,

C'était un palais infini,

Plein de bassins et de cascades

Tombant dans l'or mat ou bruni

(BAUDELAIRE, 1972, t. I, p. 101-102).

La Lisbonne sublimée d'Any where out of the world et le paysage onirique de Rêve parisien correspondent à l'idéal d'une beauté impassible et minérale, tel qu'il est défini dans la première strophe de La Beauté:

Je suis belle, ô mortels ! comme un rêve de pierre,

Et mon sein, où chacun s'est meurtri tour à tour,

Est fait pour inspirer au poète un amour

Éternel et muet ainsi que la matière

(BAUDELAIRE, 1972, t. I, p. 21).

Les commentateurs ont voulu voir dans ces " rêves de pierre " le produit de visions hallucinatoires engendrées par le hachisch ${ }^{3}$. Dans Les Paradis artificiels, Baudelaire décrit en ces termes l'ivresse créée par la drogue :

Vous savez que le haschisch invoque toujours des magnificences de lumières, des splendeurs glorieuses, des cascades d'or liquide ; toute lumière lui est bonne, celle qui ruisselle en nappe et celle qui s'accroche comme du paillon aux pointes et aux aspérités $[\ldots]^{4}$ (BAUDELAIRE, 1972, p. 418).

Caprices d'esthète, les cités de marbre et d'eau de Baudelaire s'apparentent aux fantaisies architecturales de Théophile Gautier, qui ressuscite dans Une nuit de Cléopâtre (1839) et dans Le Roman de la momie (1857) les métropoles de l'ancienne Égypte, réfléchies par les «nappes d'étain fondu » du Nil et où le vert est une " couleur inconnue " : " aucune oasis de feuillage n['y] rafraîch [i]t le regard $»^{5}$ (GAUTIER, 2011, p. 594-595). En 1851, dans un article intitulé "Paris futur ", le même Gautier développe une rêverie utopiste inspirée par les fantasmagories de Piranesi et de John Martin. Il imagine une ville aux allures babyloniennes, dominée par les éléments minéraux et aquatiques :

Je commence à tailler dans les flancs des collines lointaines des tranches gigantesques pour le soubassement des édifices ; bientôt les angles des frontons s'ébauchent dans la vapeur, les pyramides découpent leurs pans de marbre,

3 Voir la notice de Claude Pichois sur Rêve parisien ; CEuvres complètes, éd. cit., t. I, p. 1040-1041.

4 Les Paradis artificiels.

5 Une nuit de Cléopâtre (1839). 
les obélisques s'élancent d'un seul jet comme des points d'admiration de granit ; des palais démesurés s'élèvent sur des superpositions de terrasses en recul, escalier colossal, que pourraient seuls enjamber les géants du monde préadamite. Je vois s'allonger sur des colonnes trapues, fortes comme des tours et rayées de cannelures en spirales où six hommes se cacheraient, des frises faites de quartiers de montagnes et couvertes de zodiaques monstrueux, d'hiéroglyphes menaçants ; des arches de pont se courbent au-dessus du fleuve qui reluit à travers la ville qu'il tranche, comme un damas dans un col à moitié coupé ; les lacs d'eau salée, où sautent les léviathans privés, miroitent sous un rayon de lumière, et le grand cercle d'or d'Osymandias étincelle comme une roue détachée du char du soleil ! (GAUTIER, 1856, p. 372)

La végétation n'est pas absente du Paris futur, mais elle ne fait l'objet d'aucune description. Gautier se borne à indiquer que les rues sont bordées d'arbustes en fleurs - orangers, magnolias, lauriers, camélias - et que "d'immenses et nombreux squares pleins d'arbres, de fleurs et de fontaines " sont disséminés dans la ville (GAUTIER, 1856, p. 386). Dans l'œuvre de Baudelaire comme dans celle de Gautier, le végétal est banni au nom d'un " terrorisme de la forme et de la beauté ", selon l'heureuse formule de Patrick Labarthe (1999, p. 374) ${ }^{6}$. "Irrégulier» (Rêve parisien), il est l'antithèse de l'Art.

Le végétal peut cependant présenter un intérêt esthétique quand il se pare des atours de l'artifice : couleurs éclatantes, silhouette graphique, apparence bizarre. Dans Une nuit de Cléopâtre, Gautier accorde une attention particulière à la flore du désert, qui se prête à la rêverie plastique :

de loin en loin un maigre palmier s'épanouissait à l'horizon, tel un crabe végétal ; un nopal épineux brandissait ses feuilles acérées comme des glaives de bronze ; un carthame, trouvant un peu d'humidité à l'ombre d'un tronçon de colonne, piquait d'un point rouge l'uniformité générale (GAUTIER, 2011, p. 595).

De la même façon, Baudelaire nourrit une passion pour les curiosités horticoles (la " tulipe noire " et le "dahlia bleu " de L'Invitation au voyage en prose, GAUTIER, 2011, p. 303), pour les spécimens exotiques (le Yucca gloriosa de Chazal, exposé au Salon de $1845^{7}$; les " verts tamariniers " de Parfum exotique (BAUDELAIRE, 1972, t. I, p. 26.) ; les " cocotiers" du Cygne (BAUDELAIRE, 1972, p. 87) ; les « arbres bizarres et luisants » et les «mélancoliques filaos » des Projets (BAUDELAIRE, 1972, p. 314-315) et pour les compositions artistement travaillées, à l'image du « thyrse ", qui conjugue

6 Sur Baudelaire et les fantaisies architecturales de Gautier, voir : Labarthe (1999, p. 369-375).

7 Voir le Salon de 1845 (BAUDELAIRE, 1972, t. II, p. 397). 
la « ligne droite » et la « ligne arabesque " (BAUDELAIRE, 1972, p. 336). Dans L'Invitation au voyage en prose, il évoque un " pays de Cocagne ", orné de "savantes et délicates végétations", «supérieur aux autres, comme l'Art l'est à la Nature, où celle-ci est réformée par le rêve, où elle est corrigée, embellie, refondue " (BAUDELAIRE, 1972, p. 301). Il recourt ailleurs aux comparaisons végétales - florales, plus particulièrement -, dans un contexte macabre : dans Une martyre, la tête de la suppliciée repose "comme une renoncule " tandis que dans Une charogne, la " carcasse superbe " s'épanouit au soleil « comme une fleur" (BAUDELAIRE, 1972, respectivement p. 112 et 31). Dans la préface aux Euvres complètes de Baudelaire, Gautier reliera le goût du poète pour les "fleurs étranges " au "style de décadence ", à la "langue marbrée déjà des verdeurs de la décomposition et comme faisandée du bas-empire romain » et aux « raffinements compliqués de l'école byzantine » ${ }^{8}$ (GAUTIER, 1868, t. I ; GUYAUX, 2007, p. 476-477, 479).

"La haine du végétal " s'exprime déjà dans la lettre que Baudelaire adresse, à la fin de 1853 ou au début de 1854, à Fernand Desnoyers, qui lui demande de contribuer à un volume d'hommage à Claude-François Denecourt, guide attitré des promeneurs dans la forêt de Fontainebleau :

Mon cher Desnoyers, vous me demandez des vers pour votre petit volume, des vers sur la Nature, n'est-ce pas ? sur les bois, les grands chênes, la verdure, les insectes, - le soleil, sans doute? Mais vous savez bien que je suis incapable de m'attendrir sur les végétaux et que mon âme est rebelle à cette singulière religion nouvelle, qui aura toujours, ce me semble, pour tout être spirituel, je ne sais quoi de shocking. Je ne croirai jamais que l'âme des Dieux habite dans les plantes, et, quand même elle y habiterait, je m'en soucierais médiocrement et considérerais la mienne comme d'un bien plus haut prix que celle des légumes sanctifiés. J'ai même toujours pensé qu'il y avait dans la Nature, florissante et rajeunie, quelque chose d'affligeant, de dur, de cruel, - un je ne sais quoi qui frise l'impudence (BAUDELAIRE, 1972, t. I, p. 248).

Baudelaire s'élève contre les chantres de la Nature, qui se complaisent dans l'imitation naïve des grands romantiques. Il démarque le premier vers d'un poème de Victor de Laprade, À un grand arbre, recueilli dans le «Livre deuxième » («Le Poème de l'arbre ») d'un recueil intitulé Odes et poèmes (1843) :

L'esprit calme des dieux habite dans les plantes

(LAPRADE, 1878, t. I, p. 179).

8 Sur Baudelaire et l'esthétique décadente, voir GUYAUX, 2007, p. 106-121 ; Antoine Compagnon, Baudelaire devant l'innombrable, Paris, Presses de l'université Paris-Sorbonne, 2003, rééd. 2018, p. 16-19. 
Walter Benjamin s'étonnait de la « contradiction entre la théorie des correspondances naturelles et le refus de la nature» (BENJAMIN, 1982, p. 213). Jean-Christophe Bailly a montré depuis que le « renoncement à la nature ", dans l'œuvre de Baudelaire, n'a pas la « couleur du deuil » : la nature n'a procuré au poète des Fleurs du Mal que des "enchantements mineurs " (BAILLY, 2015, p. 27). Baudelaire est moins sensible à la nature qu'à sa représentation : " La nature dont il parle [...] c'est toujours celle qui fait le fond des actions humaines dans les tableaux de Delacroix ; c'est quelque chose de vert et de sombre, de dramatique et d'enlevé, c'est une puissance mais au second degré, relevée, transfigurée par l'imagination » (BAILLY, 2015, p. 27).

Si Baudelaire refuse de "s'attendrir sur les végétaux ", c'est aussi parce qu'il méprise le sentimentalisme. Sa critique du lyrisme champêtre reflète son aversion pour le pathos, qui se traduit aussi dans ses boutades contre Musset et les poètes élégiaques (" À propos du sentiment, du cour, et autres saloperies féminines, souvenez-vous du mot profond de Leconte de Lisle : "Tous les Élégiaques sont des canailles ${ }^{\natural}$ ) ou contre le misérabilisme de Victor Hugo et de ses disciples ("Tendre et profond amour du peuple ${ }^{10} ! »$ ) . "Les singes du sentiment sont, en général, de mauvais artistes ", écrit-il dans le Salon de 1846, commentant un tableau religieux d'Ary Scheffer ${ }^{11}$ (BAUDELAIRE, 1972, p. 478). Moment fondateur dans l'histoire de la poésie moderne, la rupture de Baudelaire avec le sentiment de la nature coïncide avec l'avènement de la société industrielle et d'une poésie urbaine qui explore les tourments de l'âme moderne ${ }^{12}$. Gautier formule brillamment cette idée dans la préface aux Euvres complètes de Baudelaire :

Baudelaire, s'il a le sens du grand paysage tropical où éclatent comme des rêves des explosions d'arbres d'une élégance bizarre et gigantesque, n'est que médiocrement touché par les petits sites champêtres de la banlieue; et ce n'est pas lui qui s'ébaudirait comme les philistins de Henri Heine devant la romantique efflorescence de la verdure nouvelle et se pâmerait au chant des moineaux. Il aime à suivre l'homme pâle, crispé, tordu, convulsé par les passions factices et le réel ennui moderne à travers les sinuosités de cet immense madrépore de Paris, à le surprendre dans ses malaises, ses angoisses, ses misères, ses prostrations et ses excitations, ses névroses et ses désespoirs (GAUTIER, 1868, p. 479).

9 Baudelaire à Narcisse Ancelle, 18 février 1866. BAUDELAIRE, 1972 (1973), t. II, p. 611. Voir aussi la lettre à Armand Fraisse du 18 février 1860, BAUDELAIRE, 1972, t. I, p. 675, et les BAUDELAIRE, 1972 (1973), t. II, p. 56, 110, 168, 234-235.

10 "Anniversaire de la naissance de Shakespeare ", Le Figaro, 14 avril 1864. BAUDELAIRE, 1972 (1973), t. II, p. 228.

11 Salon de 1846.

12 Sur l'avènement de la " civilisation urbaine ", voir Antoine Compagnon, Baudelaire devant l'innombrable, p. 16 ; Baudelaire l'irréductible, Paris, Flammarion, 2014, p. 213-219 ("Vers un sublime urbain »). 
La lettre de Baudelaire à Fernand Desnoyers s'inscrit par ailleurs dans un contexte polémique. L'allergie du poète aux " légumes sanctifiés » peut apparaître comme une réaction contre les croyances animistes de "l'école païenne ». Publié dans La Semaine théâtrale en janvier 1852, l'article que Baudelaire consacre à "l'école païenne ", précisément, est assez proche dans le temps de la lettre du poète à Fernand Desnoyers. Il ironise sur le retour du dieu Pan, prophétisé par les adeptes du néo-paganisme :

Il s'est passé dans l'année qui vient de s'écouler un fait considérable. [...] Dans un banquet commémoratif de la révolution de Février, un toast a été porté au dieu Pan, oui, au dieu Pan, par un de ces jeunes gens qu'on peut qualifier d'instruits et d'intelligents.

«Mais, lui disais-je, qu'est-ce que le dieu Pan a de commun avec la révolution? - Comment donc? répondait-il ; mais c'est le dieu Pan qui fait la révolution. Il est la révolution.

- D'ailleurs, n'est-il pas mort depuis longtemps ? Je croyais qu'on avait entendu planer une grande voix au-dessus de la Méditerranée, et que cette voix mystérieuse, qui roulait depuis les colonnes d'Hercule jusqu'aux rivages asiatiques, avait dit au vieux monde : LE DIEU PAN EST MORT !

- C'est un bruit qu'on fait courir. Ce sont de mauvaises langues ; mais il n'en est rien. Non, le dieu Pan n'est pas mort ! le dieu Pan vit encore, reprit-il en levant les yeux au ciel avec un attendrissement fort bizarre... Il va revenir. » Il parlait du dieu Pan comme du prisonnier de Sainte-Hélène.

"Eh quoi, lui dis-je, seriez-vous donc païen ?

- Mais oui, sans doute ; ignorez-vous donc que le Paganisme bien compris, bien entendu, peut seul sauver le monde? Il faut revenir aux vraies doctrines, obscurcies un instant par l'infâme Galiléen ${ }^{13}$ (BAUDELAIRE, 1972 (1973), t. II, p. 44).

Et l'auteur des Fleurs du Malde conclure « que cet excès de paganisme est le fait d'un homme qui a trop lu et mal lu Henri Heine et sa littérature pourrie de sentimentalisme matérialiste ${ }^{14}$ "(BAUDELAIRE, 1972 (1973), t. II, p. 45). Baudelaire fait ici allusion aux théories politico-mystiques de Henri Heine, qui, dans De l'Allemagne (1835), définit le panthéisme comme "la sanctification de la nature et la réintégration de l'homme dans ses droits divins " (HEINE, 1855, t. I, p. 173). Le " philosophe de la nature ", écrit encore Heine, est un révolutionnaire qui « se met en communication avec les pouvoirs originels de la terre » afin de conjurer « les forces cachées de la tradition» (HEINE, 1855, t. I, , p. 181).

13 "L'École païenne », La Semaine théatrale, 22 janvier 1852.

14 Voir Paolo Tortonese, «La scuola pagana esiste davvero ? Una polemica di Baudelaire », dans Il Paganesimo nella letteratura dell'Ottocento, a cura di Paolo Tortonese, Roma, Bulzoni, 2009, p. 169-186. 
$\mathrm{La}$ « haine du végétal » participe en outre de l'esprit « lycanthropique » qui traverse les poèmes du Spleen de Paris. Vers 1865-1866, Baudelaire envisageait d'intituler son recueil de poèmes en prose Petits poèmes lycanthropes, vraisemblablement en hommage à Pétrus Borel, surnommé "le lycanthrope ", à qui il avait consacré un article dans la Revue fantaisiste, le 15 juillet $1861^{15}$. Dans Champavert, contes immoraux (1833), le recueil de contes horrifiques de Borel, le rêve bucolique du retour à la terre se transforme en cauchemar. Champavert, le héros éponyme, y évoque sur un mode parodique la monotonie des saisons et des paysages de la forêt de Fontainebleau, dans laquelle il reconnaît une image de l'absurdité de l'existence :

Une seule chose me plairait moins peut-être, la monotonie, la sempiternelle physionomie de la nature : toujours de la pluie et du soleil, du soleil et de la pluie; toujours le printemps et l'automne, le chaud et la froidure ; toujours, à tout jamais. Rien n'est-il plus ennuyeux qu'une fixité, qu' une mode inamovible, qu'un almanach perpétuel. Tous les ans, des arbres verts et toujours des arbres verts ; Fontainebleau ! qui nous délivrera des arbres verts ? Que cela m’ébête !... Pourquoi, non plus de variété ? pourquoi les feuilles ne prendraient-elles pas tour à tour les couleurs de l'arc-en-ciel ? Fontainebleau ! que cette verdure est sotte! (BOREL, 2002, p. 217)

Dans le sillage de Sade, Baudelaire et Borel cultivent le même scepticisme à l'égard des idéaux philanthropiques des Lumières, sur lesquels se fonde la philosophie du progrès. Dans Champavert, les personnages qui incarnent la pureté et l'innocence subissent les pires sévices, à l'instar de Dina la belle Juive, « heureuse enfant » qui ne sait pas « que la société pervertit tout, que le fantasme de la possession et de la religion endurcit et donne la soif du sang, que l'homme bon dans l'état naturel, civilisé devient soldat, propriétaire, prêtre, juge, bourreau " ${ }^{16}$ (BOREL, 2002, p. 141). Traducteur de Robinson Crusoé, Pétrus Borel souscrit à l'idée rousseauiste de la bonté originelle de l'homme : le Mal est, à ses yeux, un produit de la civilisation. Mais il reste circonspect devant le mythe de la Nature nourricière : la Nature est impuissante à conjurer les malheurs de l'existence. Influencé par Edgar Poe et Joseph de Maistre, Baudelaire, qui croit au péché originel et à la perversité naturelle de l'homme, considère lui aussi que l'insensibilité de la Nature reflète le

15 Deux poèmes en prose de Baudelaire, La Fausse Monnaie et Le Diable [Le Joueur généreux], ont paru

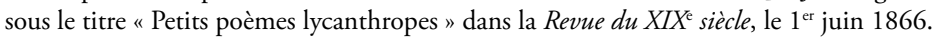

16 Sur Borel héritier de Sade, voir Mario Praz, La Chair, la mort et le diable dans la littérature française du XIX $X^{e}$ siècle (1930), traduit de l'italien par Constance Thompson-Pasquali, Paris, Denoël, 1977, rééd. Gallimard, coll. Tel, 1998, p. 132-137 ; Béatrice Didier, "Madame Putiphar, roman sadien ?", dans Pétrus Borel, Madame Putiphar, Paris, Régine Deforges, 1972, p. VII-XX ; Jean-Luc Steinmetz, Pétrus Borel. Un auteur provisoire, Villeneuve d'Ascq, Presses universitaires de Lille, 1986, p. 71-79. 
caractère implacable de la destinée. Pire, la Nature est complice du Mal qui travaille dans les replis de l'âme humaine : elle a " quelque chose d'affligeant, de dur, de cruel ", selon le mot du poète dans sa lettre à Desnoyers; elle est le théâtre des luttes assassines que se livrent les hommes. Dans Le Gâteau, deux enfants s'acharnent ainsi l'un sur l'autre, dans un paysage "d'une grandeur et d'une noblesse irrésistibles ${ }^{17}$ "(BAUDELAIRE, 1972, t. I, p. 297). La " haine du végétal " peut apparaître en ce sens comme une composante de l'antiprogressisme de Baudelaire ${ }^{18}$.

17 Sur Le Gâteau, voir Jean Starobinski, «Sur Rousseau et Baudelaire. Le dédommagement et l'irréparable », dans Le Lieu et la formule. Hommage à Marc Eigeldinger, Neuchâtel, À la Baconnière, 1978, p. 47-59; Melvin Zimmerman, "Trois études sur Baudelaire et Rousseau », dans Études baudelairiennes (Neuchâtel), IX, 1981, p. 31-71 ; Jean Starobinski, "Rousseau, Baudelaire, Huysmans (les pains d'épices, le gâteau et l'immonde tartine) ", dans Baudelaire, Mallarmé, Valéry. New Essays in Honour of Lloyd Austin, Cambridge University Press, 1982, p. 128-141 ; Marc Eigeldinger, Mythologie et intertextualité, Genève, Slatkine, 1987 ("Baudelaire juge de Jean-Jacques », p. 121-139) ; Patrick Labarthe, Baudelaire et la tradition de l'allégorie, op. cit. ("Le Gâteau : Maistre contre Rousseau ?", p. 245-251).

18 Sur l'antiprogressisme de Baudelaire, voir Théophile Gautier, préface aux Euvres complètes de Baudelaire, rééd. cit., p. 478 ; Marc Eigeldinger, Mythologie et intertextualité, Genève, Slatkine, 1987 (" Baudelaire et la mythologie du progrès ", p. 57-68) ; André Guyaux, "Le pessimisme de Baudelaire », dans Studi di letteratura e di linguistica, [Quaderno n. 6], a cura di Marina Vitale, Napoli, Edizioni scientifiche italiane, 1994, p. 69-80 ; Patrick Labarthe, "Le Spleen de Paris ou le livre des pauvres ", L'Année Baudelaire, n 5, 1999, p. 99-118 ; id., "Baudelaire et "le dieu de l'Utile ", L'Année Baudelaire, n 16, 2012, p. 83-100 ; Antoine Compagnon, Baudelaire l'irréductible, 2014 («L'horrible ville », p. 169-219). 


\section{References}

BAILLY, Jean-Christophe. La Fin de l'hymne. Paris : Christian Bourgois, 2015.

BAUDELAIRE, Charles. Euvres complètes. Texte établi, présenté et annoté par Claude Pichois. Paris : Gallimard, 1975. (Coll. Bibliothèque de la Pléiade).

BAUDELAIRE, Charles. Correspondanec. Texte établi, présenté et annoté par Claude Pichois, avec la collaboration de Jean Ziegler. Paris : Gallimard, 1972. (Coll. Bibliothèque de la Pléiade).

BENJAMIN, Walter. Zentralpark, dans Charles Baudelaire. Un poète lyrique à l'apogée du capitalisme. Paris : Payot, 1982.

BOREL, Pétrus. Champavert, le lycanthrope. Champavert, contes immoraux (1833). Texte établi d'après l'édition originale, présenté et annoté par Jean-Luc Steinmetz. Paris : Phébus, 2002.

GAUTIER, Théophile. Euvres. Édition établie Paolo Tortonese (1995). Paris : Laffont, 2011. (Coll. Bouquins).

GAUTIER, Théophile. Préface. In : BAUDELAIRE, Charles. CEuvres complètes. Paris : Michel Lévy, 1868.

GAUTIER, Théophile. Caprices et zigzags (1852). Paris : Hachette, 1856.

GUYAUX, André. Baudelaire. Un demi-siècle de lectures des "Fleurs du Mal » (1855-1905). Paris : Presses de l'université Paris-Sorbonne, 2007.

HEINE, Henri. La religion et la philosophie en Allemagne. De l'Allemagne (1835). Paris : Michel Lévy, 1855.

LABARTHE, Patrick. Baudelaire et la tradition de l'allégorie. Genève : Droz, 1999. LAPRADE, Victor de. Odes et poèmes (1843). CEuvres poétiques. Paris : Lemerre, 1878.

MERSON, Olivier. Lisbonne : histoire, monuments, mours. Paris : Hachette, 1857.

VOGEL, Charles. Le Portugal et ses colonies. Paris : Guillaumin, 1860.

Aurélia Cervoni. Doutora pela universidade de Paris-Sorbonne, onde ocupa um posto de pesquisadora. Autora de um livro sobre Théophile Gautier (Théophile Gautier devant la critique, 1830-1872, Classiques Garnier, 2016). Colaborou com a edição das Euvres complètes de Rimbaud publicadas na Pléiade (2009), dirigida por André Guyaux, e editou, com Andrea Schellino, Le Spleen de Paris de Baudelaire (GF-Flammarion, 2017).

E-mail: aurelia.cervoni@sorbonne-universite.fr 